\title{
On a Correlation between the Ionospheric Electric Field and the Time Derivative of the Magnetic Field
}

\author{
R. R. Ilma, ${ }^{1}$ M. C. Kelley, ${ }^{1}$ and C. A. Gonzales ${ }^{2}$ \\ ${ }^{1}$ School of Electrical and Computer Engineering, Cornell University, Ithaca, NY 14853, USA \\ ${ }^{2}$ IBM Thomas J. Watson Research Center, Yorktown Heights, NY 10598, USA \\ Correspondence should be addressed to R. R. Ilma, rri5@cornell.edu
}

Received 11 November 2011; Accepted 20 February 2012

Academic Editor: Yuichi Otsuka

Copyright $(2012$ R. R. Ilma et al. This is an open access article distributed under the Creative Commons Attribution License, which permits unrestricted use, distribution, and reproduction in any medium, provided the original work is properly cited.

\begin{abstract}
A correlation of the ionospheric electric field and the time derivative of the magnetic field was noticed over thirty years ago and has yet to be explained. Here we report on another set of examples during the superstorm of November 2004. The electric field in the equatorial ionosphere, measured with the Jicamarca incoherent scatter radar, exhibited a $3 \mathrm{mV} / \mathrm{m}$ electric field pulse that was not seen in the interplanetary medium. It was, however, accompanied by a correlation with the time derivative of the magnetic field measured at two points in Peru. Our inclination was to assume that the field was inductive. However, the time scale of the pulse was too short for the magnetic field to penetrate the crust of the Earth. This means that the area threaded by $\partial B / \partial t$ was too small to create the observed electric field by induction. We suggest that the effect was caused by a modulation of the ring current location relative to the Earth due to the electric field. This electric field is required, as the magnetic field lines are considered frozen into the plasma in the magnetosphere. The closer location of the ring current to the Earth in turn increased the magnetic field at the surface.
\end{abstract}

\section{Introduction}

In his Ph.D. thesis, Gonzales [1] published Figure 1 using interplanetary and auroral indices, electric field measurements using the incoherent scatter radar (ISR) technique at Chatanika (Alaska) and Jicamarca (Peru), and $\partial B / \partial t$ measured on the ground at San Juan, Puerto Rico. The correlation between the latter three parameters was excellent. The ratio $E /(\partial B / \partial t)$ was 50 million meters. Here we report on an extensive set of observations we believe to be of the same type obtained during the superstorm of November 2004 (see [2], and companion papers).

\section{Data Presentation}

Various unusual phenomena that occurred in the November 2004 magnetic storm are documented in a series of papers, the first of which summarizes many of the observations from the interplanetary medium to the equatorial ionosphere [2]. The second part of the two-phase storm was monitored by the ISR chain in the American sector with unprecedented coverage. At Jicamarca, Peru, the eastward electric fields can be deduced from ISR drifts obtained from pulse-to-pulse experiments when the transmitting antenna was pointed perpendicular to the geomagnetic field $\mathbf{B}$ [3]. The radial velocity is a measurement of F-region vertical $\mathbf{E} \times \mathbf{B}$ drift with typical time and range resolutions of 5 minutes and $15 \mathrm{~km}$, respectively. The uncertainty of these ion drifts is less than $1 \mathrm{~m} / \mathrm{s}$. In addition to the observed electric field, the horizontal component $(\mathrm{H})$ of the geomagnetic field was measured at the stations indicated in Table 1 and included in the analysis. The magnetic latitudes of these stations were calculated using the International Geomagnetic Reference Field (IGRF) magnetic field model. these stations are located in the American Sector. The Jicamarca magnetometer provides measurements every second with an uncertainty of $0.1 \mathrm{nT}$ and the Piura magnetometer every 10 seconds with $1 \mathrm{nT}$ of resolution [4]. From all magnetic stations, the 1-minute average of measurements was utilized. Finally, to consider a magnetospheric perspective, the parallel component of the magnetic field measured by the geostationary satellite GOES 12 is also used in this study. 
TABLE 1: Magnetometer stations.

\begin{tabular}{lcccc}
\hline Station name & Location & Geographic longitude, deg & Geographic latitude, deg & Magnetic latitude, deg \\
\hline Jicamarca & Peru & 283.13 & -11.95 & -1.73 \\
Piura & Peru & 279.36 & -5.18 & 4.96 \\
College & Alaska & 212.14 & 64.87 & 65.38 \\
\hline
\end{tabular}

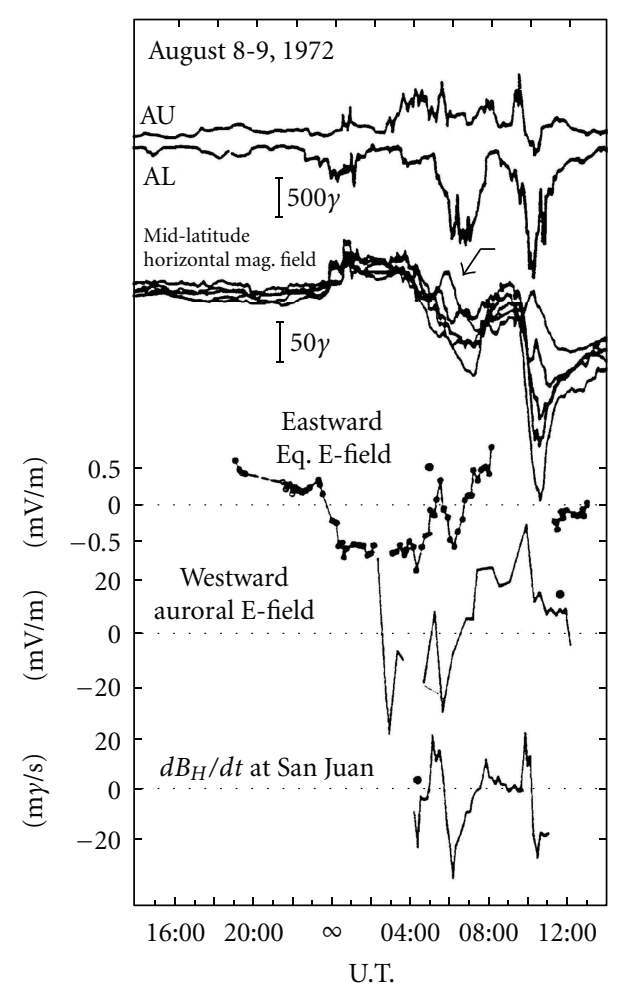

Figure 1: From top to bottom: The AU, AL indices; five superposed midlatitude magnetograms (Kakioka, Tashkent, Tangerang, San Juan, Honolulu) with the arrow pointing to the San Juan magnetogram; the zonal electric field at the equator (Jicamarca, Peru); the auroral zonal electric field at College, Alaska; the time derivative of the horizontal component of the magnetic field at San Juan, Puerto Rico. In the last three panels, the dots indicate local magnetic midnight. (After [5]. Reproduced with permission of the American Geophysical Union).

\section{Data Analysis}

In the November 2004 event, the interplanetary electric field was correlated at a level of $85 \%$ with the zonal electric field measured at the Jicamarca Radio Observatory (JRO) [2]. The basic result is presented in Figure 2 where the delayed interplanetary electric field [6] is plotted, along with the zonal component of the electric field measured in the $\mathrm{F}$ region over JRO. The seeming lack of correlation after 0500 UT on 10 November 2004 is due to the local time dependence of the penetration electric field [6]. As was shown by [7], this deviation is expected theoretically and, in fact, shows that the two fields continue to be highly anticorrelated if local time is taken into consideration. But of interest here is the huge spike in the JRO electric field at

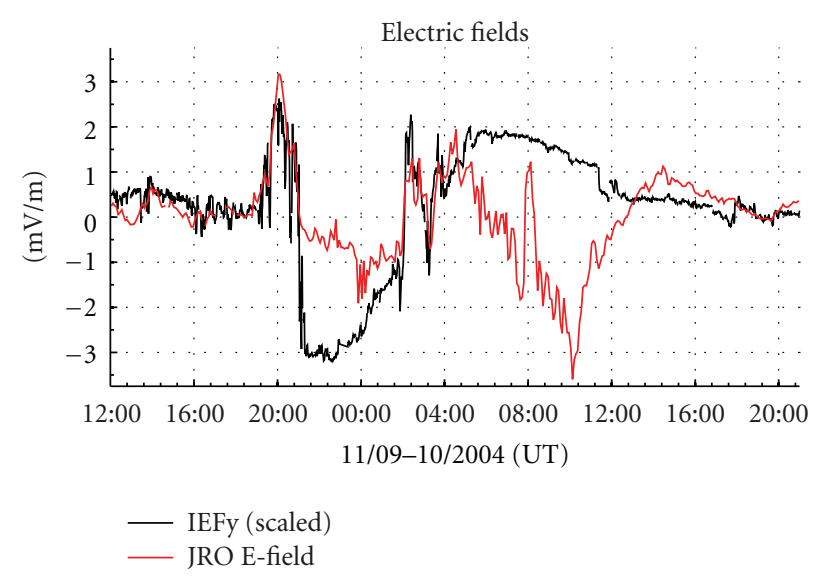

FIGURE 2: The black-line plot is the $y$-component of the electric field in the Earth's frame of rest delayed by the transit time of the solar wind to the front of the magnetosphere divided by ten for a $33 \mathrm{~h}$ period on November 2004. The red-line plot is the zonal component of the electric field in the equatorial ionosphere. (After [8]. Reproduced with permission of the European Geoscience Union).

0800 UT. The change in the electric field is $3.0 \mathrm{mV} / \mathrm{m}$, which, by equatorial standards, is a very large field. Curiously, there is no corresponding interplanetary electric field change. We conclude that this was due to a magnetospheric source.

In Figure 3, we compare the JRO electric field with the poleward component of the magnetic field measured on the ground at two locations in Peru: at Jicamarca and at Piura, about $1000 \mathrm{~km}$ north. The magnetic fields at Jicamarca and Piura are 90 degrees out of phase with the JRO electric field during the time of the large electric field change, as is clearly seen in Figure 5. In Figure 4, we plot the electric field along with the time derivative of the Jicamarca magnetic field. During the time period of 0720-0820 UT, which includes the largest electric field pulse, the two are in phase. The period of the pulse is about an hour. At other times, the fluctuations occur at higher frequencies and the phase shift is variable. During the period of 0700-0830 UT, the correlation between the JRO electric field and the time derivative of the local magnetic field is $71 \%$. The peak time derivative of the magnetic field is $6 \mathrm{nT} / \mathrm{min}$.

Figure 5 shows that the electric field and the magnetic field are 90 degrees out-of-phase. The large electric field pulse begins at $0740 \mathrm{UT}$. At 0800, GOES registered a dipolarization (see Section 4 for a definition) event in which the parallel component of $B$ increased by over $40 \mathrm{nT}$. The time delay observed in the satellite data likely is due to the location of GOES relatively near the Earth. The electric field essentially 


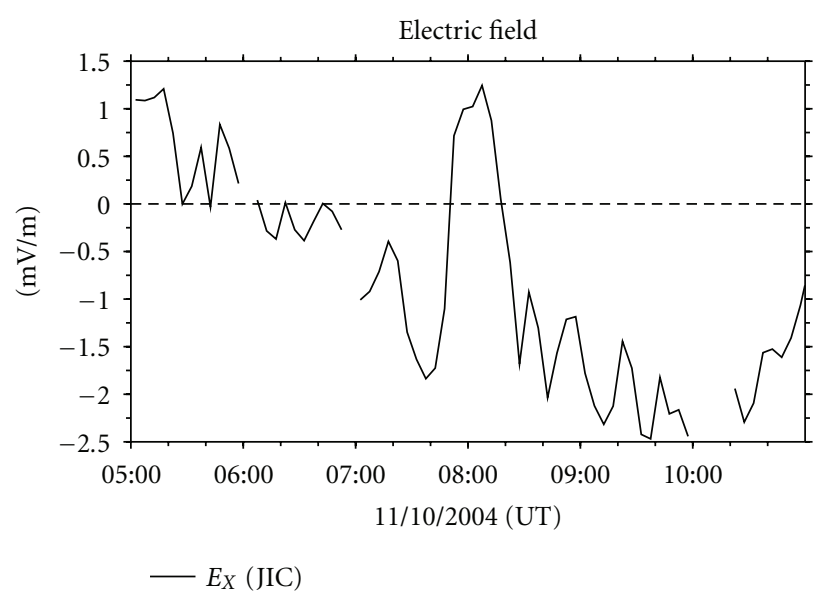

(a)

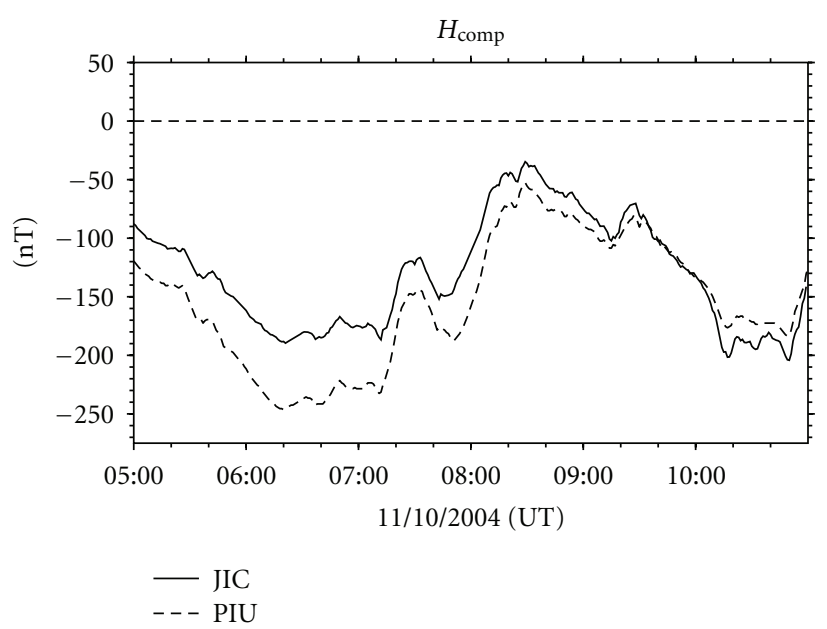

(b)

Figure 3: The eastward electric field at Jicamarca for a $6 \mathrm{hr}$ period on 10 November 2004. (b) shows the horizontal magnetic field on (Jicamarca) and off (Piura) the equator.

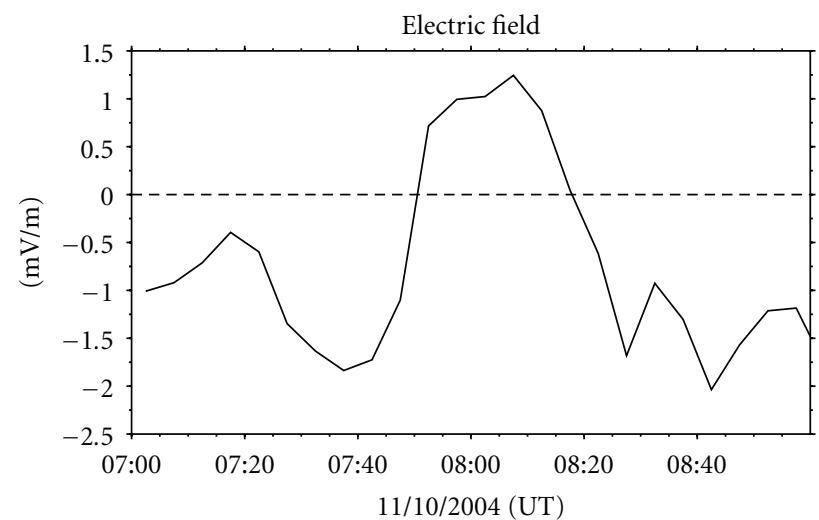

(a)

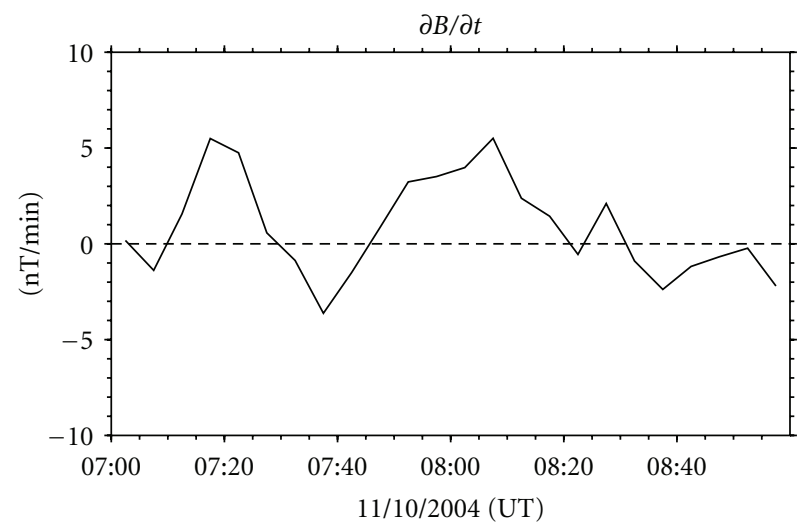

(b)

Figure 4: Comparison of the time-derivative of the horizontal component of the magnetic field simultaneously with the eastward electric field. Both quantities were measured at Jicamarca, Peru.

penetrates instantaneously [6]. In Figure 6, we investigate the $75^{\circ} \mathrm{W}$ GOES magnetic field component perpendicular to the equatorial plane.

\section{Discussion}

Maxwell's equations involve partial time and space derivatives, as opposed to the total time derivatives, which include advective terms. Comparison of the Jicamarca and equatorial magnetic fields indicates that the magnetic field of the most importance is northward at the nighttime equator. Using the integral form of Maxwell's equation, $\oint E \cdot d l=\iint(\partial B / \partial t) d s^{2}$, and assuming that the magnetic field entirely penetrates the Earth, $\partial B / \partial t \sim 0.07 \mathrm{nT} / \mathrm{s}$, and so we can estimate the induced electric field from

$$
2 \pi R_{E} E=\pi R_{E}^{2} \frac{\partial B}{\partial t}
$$

and hence,

$$
E=\frac{\partial B}{\partial t} \frac{R_{E}}{2}=0.2 \mathrm{mV} / \mathrm{m}
$$

which underestimates $E$ by a factor of ten for the November 2004 event. However, this result requires the external magnetic field to penetrate the entire Earth. Penetration phenomena are referred to as magnetic field diffusion and are described by

$$
\frac{\partial B}{\partial t}=\frac{\eta}{\mu_{0}} \nabla^{2} B,
$$

where $\eta$ is the resistivity of the medium. The time constant for penetrating the magnetic field by a distance of $L$ is thus the order of

$$
\tau=\frac{\mu_{0} L^{2}}{\eta} .
$$




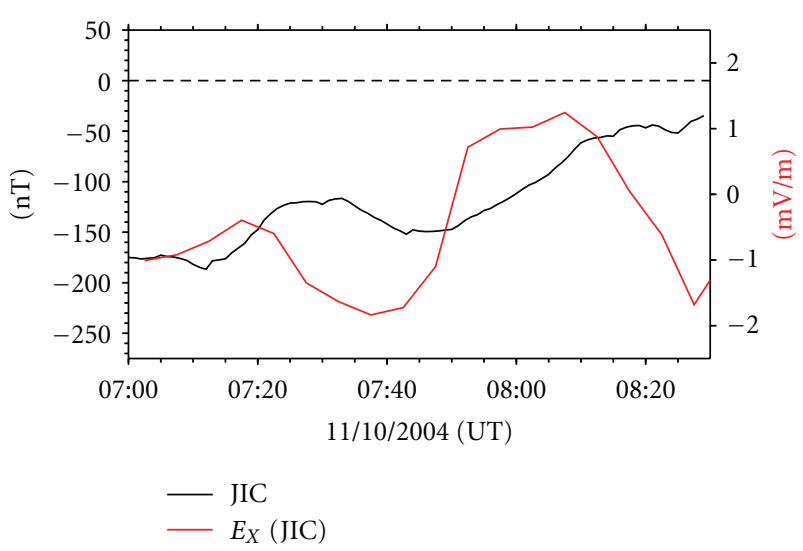

FIGURE 5: The electric and magnetic field time-series, 90 degrees out of phase over Jicamarca, Peru.

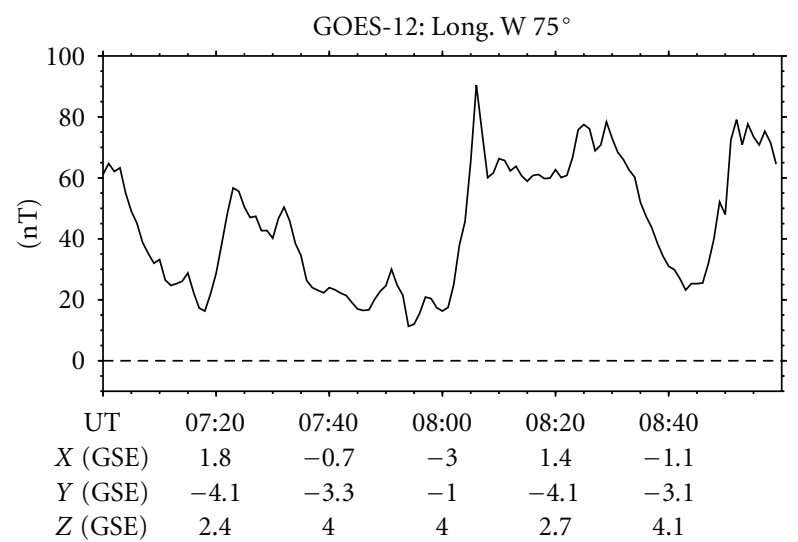

Figure 6: The GOES-12 (Long. W 75) parallel component of the magnetic field versus universal time (UT) and GSE coordinates.

Using typical crustal values of $\eta=100 \Omega / \mathrm{m}$, we find that it takes 100 hours to penetrate $1 R_{E}$ into the planet. Thus, the line integral of the electric field around the Earth at F-region heights must be equal to the surface integral of $\partial B / \partial t$ in the area between, say, $H=300 \mathrm{~km}$ and the surface. Thus, we have

$$
2 \pi R_{E} \sim(2 \pi R) H \frac{\partial B}{\partial t},
$$

where we have approximated the area enclosed by the line integral. Substituting values and solving for $E$ yields only $0.04 \mathrm{mV} / \mathrm{m}$, which is almost two orders of magnitude too small. It was tempting, of course, to treat the electric field as an inductive effect, but the numbers just do not add up. As shown above, the solid earth is a good enough conductor that, on the time scale of the pulse, it cannot even penetrate the crust.

So what is happening? Without a penetrating electric field from the IMF, we could posit an electric field generated in the magnetosphere, say, during a magnetic substorm. In Figure 7, we compare the magnetic field measured in Alaska with the electric field in Peru for the event. Reference [9] showed that the key link between perturbations in the

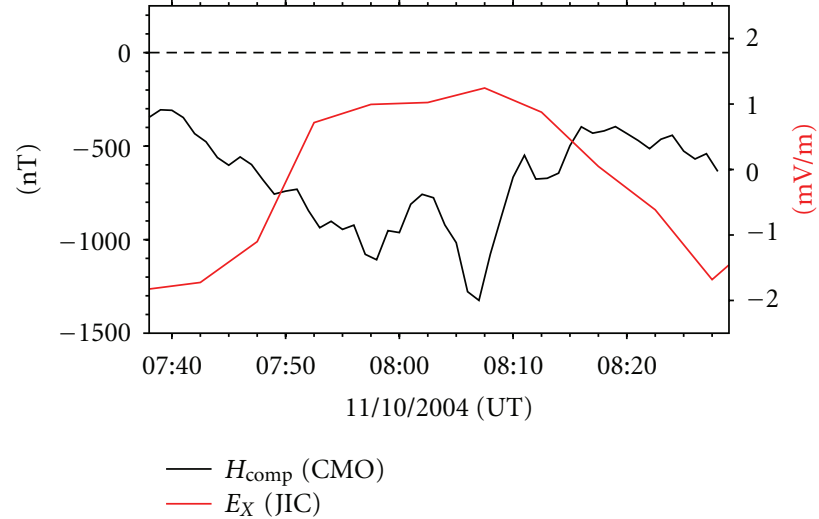

FIgURE 7: Time-derivative of the horizontal component of the magnetic field at the polar zone (College, Alaska; black solid curve) simultaneously with the eastward component of the electric field in the equatorial ionosphere (Jicamarca, Peru; red curve).

equatorial F-region electric fields and the interplanetary magnetic field is the electric field of magnetospheric origin. If there is a substorm-related, magnetospherically induced electric field oriented from dusk to dawn across the nightside, the ring current will move closer to the Earth, causing an increase in the magnetic field at the surface that will be proportional to the distance to the ring current. Since distance is the integral of the velocity and the electric field, and if the magnetospheric field penetrates to the equator, as it must, $E$ and $\partial B / \partial t$ will be correlated. During a substorm, magnetic energy stored in the distorted magnetosphere is released into the atmosphere as accelerated electrons and Joule heating. At such a time, the magnetic field relaxes towards dipole, which is the lowest energy state. This is called dipolarization, which is associated with the release of stored magnetic energy into the ionosphere-atmosphere system. Such an event was reported by [10] using the THEMIS instrumentation. Since the magnetic field lines can be considered to be frozen into the plasma, an eastward electric field must be associated with this dipolarization. They found that the equatorial magnetospheric electric field was in the range of $2-6 \mathrm{mV} / \mathrm{m}$. Observations of penetrating electric fields (PPE) at the equator showed that the PPE is at least 0.5 times the equatorial field [11]. The magnetic field at the center of a current loop is inversely proportional to the radius $a$, that is, $B=K I / a$. Thus,

$$
\frac{\partial B}{\partial t}=-K I \frac{1}{a^{2}} \frac{\partial a}{\partial t}=B \frac{1}{a} \frac{\partial a}{\partial t} .
$$

If $B=100 \mathrm{nT}, a=R_{e}$, and $\partial a / \partial t=E / B_{0}=2 \mathrm{~km} / \mathrm{s}$, then $\partial B / \partial t=2 \mathrm{nT} / \mathrm{min}$. These parameters are conservative since a strong ring current could locate at $a=3 R_{e}$ and produce $400 \mathrm{nT}$ at Earth. This mechanism seems to explain the observations. It is well known that inductive electric fields create problems for electric power systems (see [12], and references therein). We suggest that the time-varying electric fields potential reported here could be a source of power system disruptions at middle and low latitudes. 


\section{Acknowledgments}

The authors gratefully acknowledge the Jicamarca staff for providing radar and magnetic data. Magnetic data at College, Alaska, was obtained from the International Real-time Magnetic Observatory Network (INTERMAGNET) database. Magnetic data from GOES 12 were obtained from the Space Physics Interactive Data Resource (SPIDR) database. M. Kelley and R. Ilma were supported by the National Science Foundation (NSF) under Grant ATM-0551107. The Jicamarca Radio Observatory is a facility of the Geophysical Institute of Peru and is operated with support from the NSF Cooperative Agreement ATM-0432565 through Cornell University.

\section{References}

[1] C. A. Gonzales, Electric fields in the low latitude ionosphere and their relationship to magnetospheric and interplanetary phenomena, Ph.D. thesis, Cornell University, Ithaca, NY, USA, 1979.

[2] M. C. Kelley, R. R. Ilma, M. Nicolls et al., "Spectacular lowand mid-latitude electrical fields and neutral winds during a superstorm," Journal of Atmospheric and Solar-Terrestrial Physics, vol. 72, no. 4, pp. 285-291, 2010.

[3] E. Kudeki, R.F. Woodman, and Z. Feng, "Incoherent scatter radar plasma density measurements at Jicamarca using a transverse-mode differentialphase method," Geophysical Research Letters, vol. 30, no. 1255, p. 4, 2003.

[4] D. Anderson, A. Anghel, J. Chau, and O. Veliz, "Daytime vertical $\mathrm{E} \times \mathrm{B}$ drift velocities inferred from ground-based magnetometer observations at low latitudes," Space Weather, vol. 21, no. 11001, p. 9, 2004.

[5] C. A. Gonzales, M. C. Kelley, B. G. Fejer, J. F. Vickrey, and R. F. Woodman, "Equatorial electric fields during magnetically disturbed conditions. II-implications of simultaneous auroral and equatorial measurements," Journal of Geophysical Research, vol. 84, pp. 5803-5812, 1979.

[6] M. C. Kelley and E. Dao, "On the local time dependence of the penetration of solar wind-induced electric fields to the magnetic equator," Annales Geophysicae, vol. 27, no. 8, pp. 3027-3030, 2009.

[7] R. W. Nopper and R. L. Carovillano, "Polar-equatorial coupling during magnetically active periods," Geophysical Research Letters, vol. 5, pp. 699-702, 1978.

[8] M. C. Kelley, R. R. Ilma, and G. Crowley, "On the origin of prereversal enhancement of the zonal equatorial electric field," Annales Geophysicae, vol. 27, pp. 2053-2056, 2009.

[9] M. C. Kelley, B. G. Fejer, and C. A. Gonzales, "An explanation for anomalous equatorial ionospheric electric fields associated with a northward turning of the interplanetary magnetic field," Geophysical Research Letters, vol. 6, pp. 301-304, 1979.

[10] A. Runov, V. Angelopoulos, M. I. Sitnov et al., "THEMIS observations of an earthward-propagating dipolarization front," Geophysical Research Letters, vol. 36, no. 14, Article ID L14106, 2009.

[11] C. A. Gonzales, M. C. Kelley, R. A. Behnke, J. F. Vickrey, R. Wand, and J. Holt, "On the longitudinal variations of the ionospheric electric field during magnetospheric disturbances," Journal of Geophysical Research, vol. 88, pp. 9135-9144, 1983.

[12] J. G. Kappenman, "An overview of the impulsive geomagnetic field disturbances and power grid impacts as 130 sociated with the violent Sun-Earth connection events of 29-31 October 2003 and a comparative evaluation with other contemporary storms," Space Weather, vol. 3, article S08C01, 2005. 

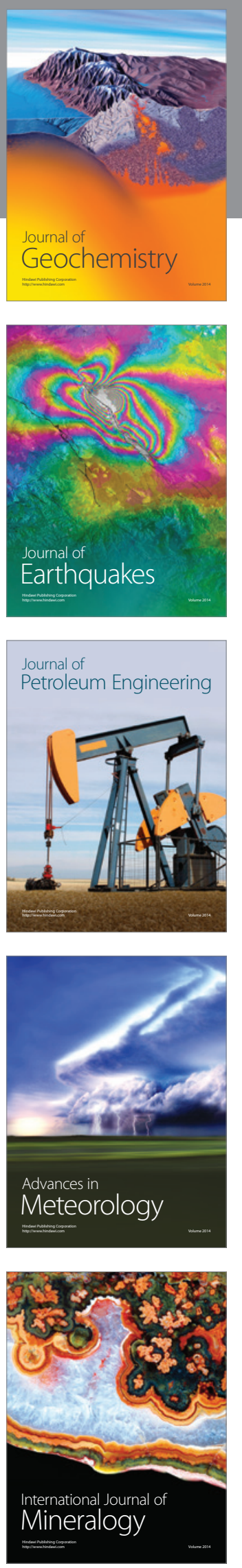
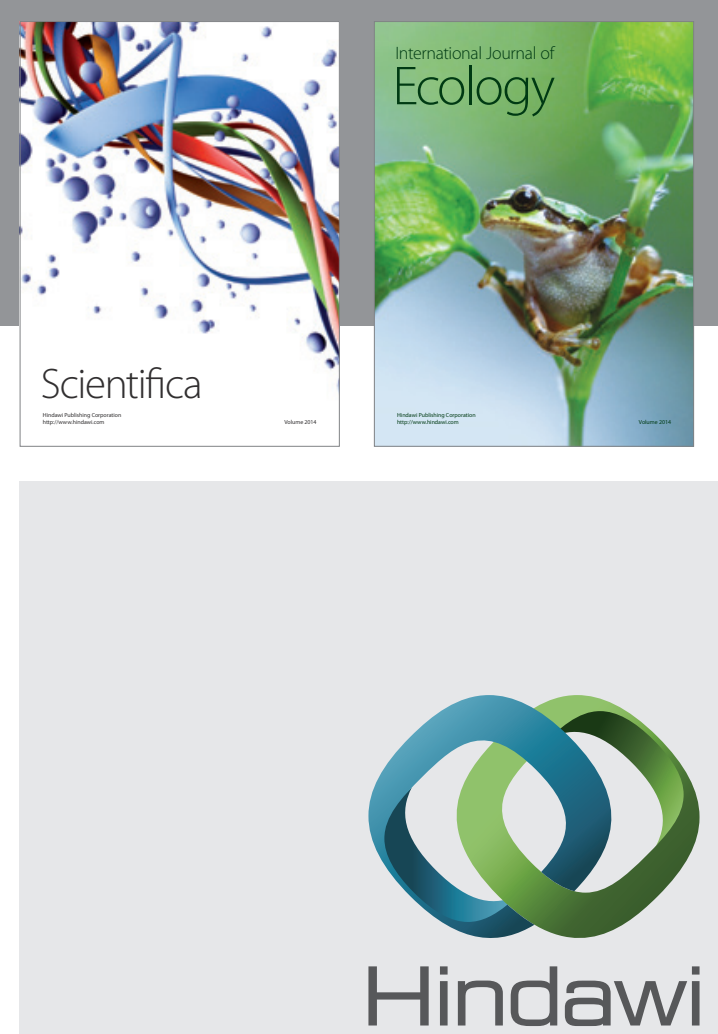

Submit your manuscripts at http://www.hindawi.com
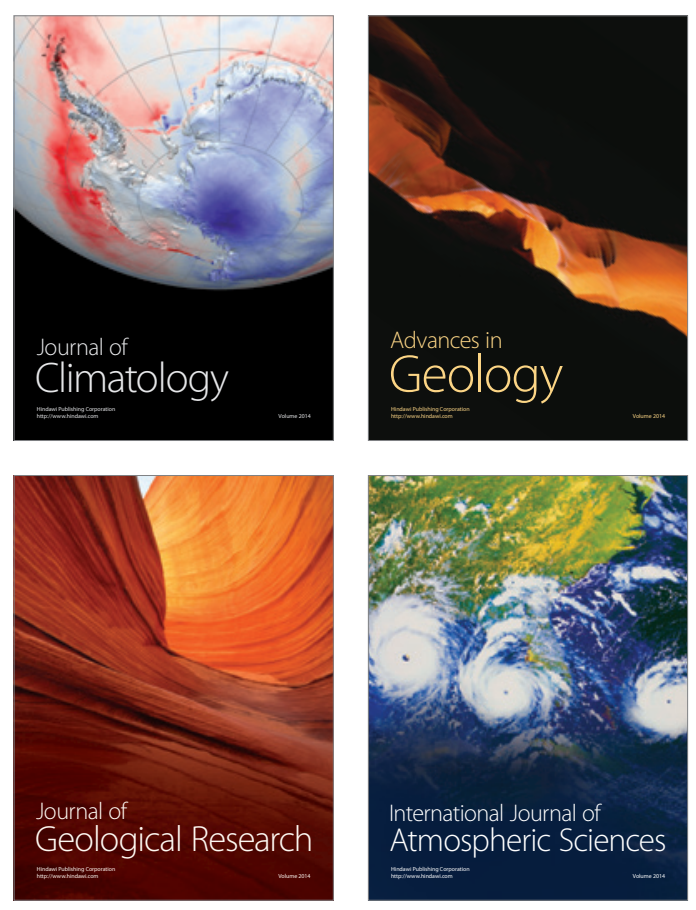
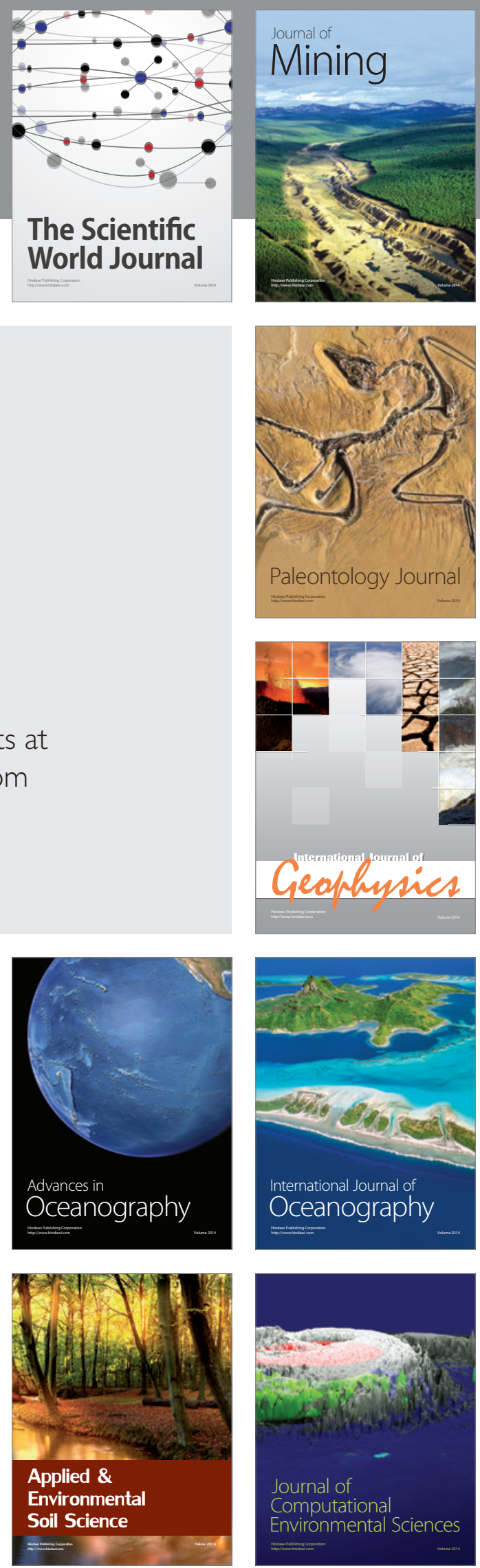